\title{
Regression Analysis to Predict Student Electric Circuits Performance
}

\section{Dr. Matthew Garett Young, Arkansas Tech University}

Matthew G. Young received his B. Sc. in Electrical Engineering from Arkansas Tech University in 2009. He participated in an NSF IREU focusing on antenna design in the summer of 2009 before obtaining his M. Sc. in Microelectronics-Photonics at the University of Arkansas in 2012. For his M. Sc. studies, he focused on the growth of silicon nanowires via plasma enhanced chemical vapor deposition. In August of 2016, he joined the faculty at Arkansas Tech University as an Assistant Professor of Electrical Engineering. His Ph.D. was completed at the University of Arkansas in May 2017. At Arkansas Tech University, Matthew is focused on establishing research experiences in photovoltaics for undergraduate and graduate students and investigating new methods to enhance engineering education in the classroom.

\section{Dr. Edward Carl Greco Jr., Arkansas Tech University}

Dr. Greco is a Professor of Electrical and Computer Engineering with research interest in biomedical signal processing. He teaches courses in digital systems, signals and systems, communications and biomedical signal processing.

Dr. Scott Marks Jordan, Arkansas Tech University

Dr. Thomas Galen Limperis, Arkansas Tech University 


\section{Regression Analysis to Predict Student Electric Circuits Performance}

Introduction

The ability to predict engineering students' future performance based upon previous academic performance would be a useful tool for identifying at-risk students and increasing retention in engineering programs. One factor in student persistence in engineering programs relates to previous course performance [1]. Many courses offered in engineering programs occur in specific sequences such that one course can have several prerequisites. An analysis of prerequisite course performance can be useful for predicting students' future performance [2]. In fact, studies have shown that pre-college academic performance can be a predictor of program graduation [3]. Although many factors have been reported to contribute to the successful completion of an engineering degree, a model has not been developed to allow at-risk students to be identified for early intervention. The objective of this study was to discover the parameters that are most sensitive and predictive of success in a key course in the electrical engineering degree program at our university and to utilize these parameters to construct such a model.

Any engineering discipline requires several semesters worth of calculus-based mathematics and physics prerequisites. It is obvious to hypothesize that the academic performance of students during the first few semesters of their college career must have an impact on their future performance through calculus classes. In fact, studies have shown that Calculus I and Calculus II can be strong indicators of future academic performance [4,5]. Others have found that other mathematics courses are key predictors, such as Linear Algebra [6]. These studies tended to focus primarily on academic factors, without considering broader implications of a student's journey to completion of their degree program. A student's "sense of belonging" has been shown to influence whether students leave engineering programs [7]. Other non-academic factors have also been shown to influence the ability of students to earn degrees including social cognitive factors such as self-efficacy [8]. Studies showing how important the predictive behavior of fundamental electrical engineering courses are to student success within their degree programs are harder to find.

Electric Circuits I is a fundamental course in an electrical engineering program. This course provides students with knowledge of essential analytical skills that apply to problems both in and out of the discipline. Such concepts taught include but are not limited to Kirchhoff's Current and Voltage Laws, Nodal and Loop Analysis, Superposition, Thevenin's Theorem and Maximum Power Transfer, and transient behavior of circuits including resistors, capacitors, and inductors.

Prediction of success in Electric Circuits I must be dependent upon the prerequisites to Electric Circuits I that typically includes a sequence in Calculus I and Calculus II. In this paper, we utilize multiple analysis techniques to explore the extent to which certain academic factors could serve as predictors of performance in an Electric Circuits I course. Both cognitive and noncognitive factors are considered, however, mostly cognitive factors seem to be optimal predictors of Electric Circuits I success. If students are successful in their first 4-semesters of a program, it is likely that they will continue to graduation. Therefore, it is important to develop a model that is useful in identifying at-risk students enrolled in an Electric Circuits I course. If a student is 
found to be at-risk based upon their Electric Circuits I grade, appropriate interventions could be applied that might increase student success. For instance, an assigned mid-semester review course was found to be effective for grade recovery for Calculus I students struggling with a D or $\mathrm{F}$ at midterm [9]. The results of this study indicate some sort of similar intervention might be appropriate for those students taking Electric Circuits I and pursuing an electrical engineering degree.

\section{Model Information}

We used the statistical language $\mathrm{R}$ to produce an ordinal logistic regression of predicting Electric Circuits I success given several factors, a correlation analysis of the same factors, and a generalized linear model $(\mathrm{glm})$ for determining graduation probability for students using data obtained from institutional records at our university. We examined available cognitive and noncognitive factors that might impact student performance in Electric Circuits I. Table 1 provides a listing of the entire set of factors that were considered in the model. ACT Scores means that ACT Math and ACT Composite scores were included. Adjusted Cumulative GPA refers Cumulative GPA for the student including the semester the student was registered in Electric Circuits I with the Circuits I grade excluded. This provides an adjusted GPA without the influence of the Electric Circuits I grade.

Our university is an undergraduate-focused university that offers engineering degrees in mechanical, electrical and computer disciplines. Within the engineering disciplines the mechanical engineering has 356 total enrolled (328 full time and 28 part-time) majors, electrical engineering program has 148 total enrolled (128 full time and 20 part-time) majors, and computer engineering has 16 total enrolled (15 full time and 1 part-time) students as of 2018.

The engineering programs at the university (computer, electrical, mechanical, and engineering physics) along with physics take a common set of core math (calculus and differential equations) and science (physics and chemistry) courses in their freshmen and sophomore years. In addition, these degree programs take the two-semester electric circuits course sequence (Electric Circuits I and II) offered by the electrical engineering department. Computer and electrical engineering majors take them in their third and fourth semester, while mechanical engineering majors schedule them in the fourth and fifth semesters and physics and engineering physics require them in their sixth and seventh semesters. Our analysis examines the first several semesters of a student's academic career which are in common with all these programs and include the prerequisite Calculus I \& II courses.

Table 1. Details of factors that were considered for use in the regression model and correlation analysis. HS $=$ High School.

\begin{tabular}{|c|c|c|}
\hline \multicolumn{3}{|c|}{ Factors Examined for Used in Ordinal Logistic Regression Analysis } \\
\hline Cognitive - College & Cognitive - Pre-College & Non-Cognitive \\
\hline Calculus I Earned Grade & HS Graduation Date & Age \\
\hline Calculus II Earned Grade & HS Rank & Gender \\
\hline Electric Circuits I Earned Grade & HS Class Size & Ethnicity \\
\hline Years to Graduation & HS Percentile & \\
\hline Adjusted Cumulative GPA & ACT Scores & \\
\hline
\end{tabular}


The model included 845 students registered in Electric Circuits I over a period of eight years. The total model student population was $9.23 \%$ female, and the students' ethnicities included 74.2\% Caucasian, 15.86\% International - Non-Resident Alien, 4.14\% Hispanic, 2.60\% African American, $1.90 \%$ Asian or Native Hawaiian-Pacific Islander, and 1.30\% American Indian. The students' majors when registered in Electric Circuits I was the following: 308 students majoring in electrical engineering, 483 mechanical engineering, and 54 majoring in other disciplines (the majority were physics or engineering physics majors). There were 767 male and 78 female students in the study.

Since some students take advanced placement (AP) exams in their pre-college academic careers, it was necessary to create a grade map so the model would function properly. Table 2 provides details on the grade map used in the model. The column, "Letter Grade Used in Model" provides the letter grade, input as ordinal data into the model. The column "Mapped Grades" provides how non-standard grades mapped to the ordinal data in the model.

Table 2. Mapping of letter grades used in the ordinal model. TA = "Transfer A", TB = "Transfer B", AP4 = "a score of 4 on the AP exam", TC = "Transfer C", "CE = C Equivalent", "AP3 = "a score of 3 on the AP exam", TD = "Transfer D", TF = "Transfer F", W = "Withdraw", TW = "Transfer Withdraw", and I = "Incomplete".

\begin{tabular}{|c|c|}
\hline Letter Grade Used in Model & Mapped Grades \\
\hline A & TA \\
\hline B & TB, AP4 \\
\hline C & TC, CE, AP3, AP \\
\hline D & TD \\
\hline F & TF, W, TW, I \\
\hline
\end{tabular}

A correlation analysis was first performed on the set of factors detailed in Table 1. This correlation analysis was used to gather some idea about how the input factors to the logistic regression were related to each other. The package corrplot was used to implement this in the $\mathrm{R}$ language [10]. A proportional odds logistic regression (polr from the MASS package [11]) was performed on the data with earned grades in Circuits I as a response and various other data (earned Calculus I grades, Adjusted Cumulative GPA, ACT scores, etc.) as a linear predictor. A Type III ANOVA analysis was applied to the output of the regression. A single predictor was utilized for each model run. For the glm model, the R package rapportools was utilized with the function glm [12]. The data was randomly partitioned into training and testing sets and a receiver operating characteristic (ROC) analysis was performed to assess the classification ability of the most optimal set of parameters in the model.

Correlation Between Factors

Figure 1 shows two different correlation matrices (A) and (B). The correlation matrix labeled (A) provides the output of correlation analysis for college-level factors used in the logistic regression. The correlation matrix labeled (B) provides the output of correlation analysis for high-school level factors used in the logistic regression. The color scale on the right-hand side of the matrices (A) and (B) provides for how strongly correlated a given set of factors are. The deep blue color represents a strong positive correlation whereas the deep red color represents a strong negative correlation. For instance, in examining (B) HS_GPA has a strong positive 
correlation with the factor HS_PERCENTILE. However, the factor HS_CLASS_SIZE is essentially independent of all factors except HS_RANK for which it shares a moderate positive correlation.

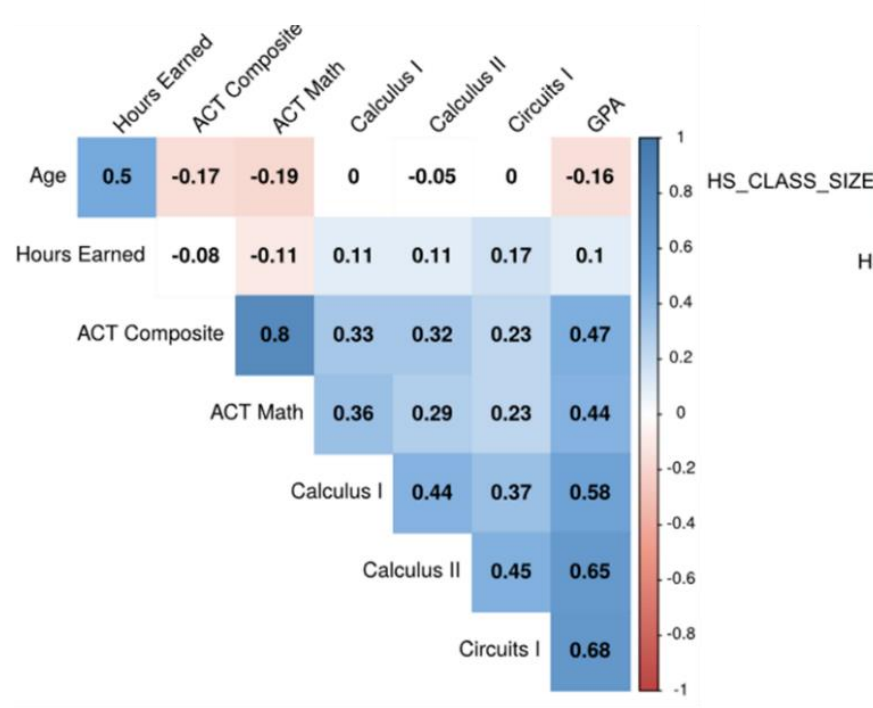

(A)

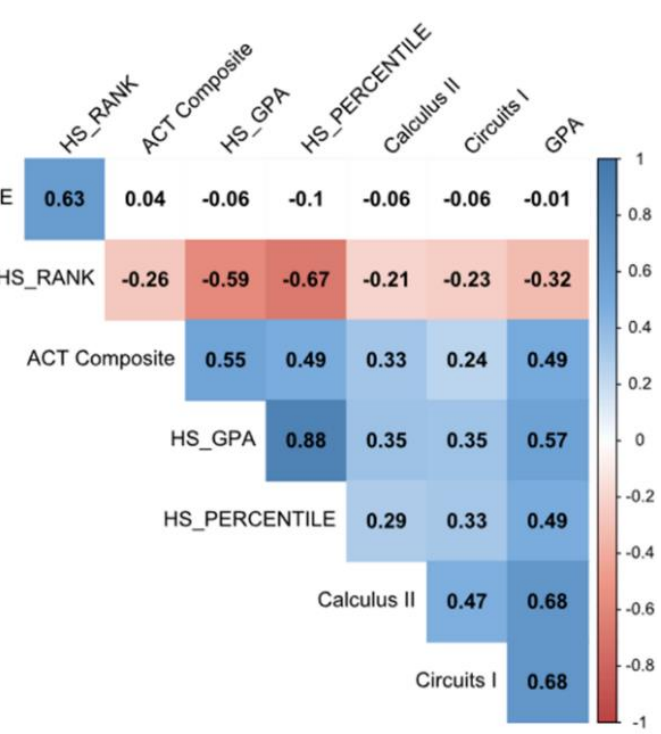

(B)

Figure 1. Correlograms showing a correlation between factors that were run in the model. HS $=$ High School and GPA $=$ Grade Point Average .

The correlation matrices provide evidence of those factors that show stronger correlations with earned grades in Circuits I. The strongest correlation for earned Circuits I grades is with adjusted cumulative GPA, while Calculus II grades, Calculus I grades, ACT Math, and ACT Composite scores show a weak positive correlation with Circuits I grades. Factors such as Age and Hours Earned show very little to no correlation with Circuits I grades.

A weak positive correlation was observed between Electric Circuits I grades and both HS_GPA (High School GPA) and HS_PERCENTILE (High School Percentile). It can also be observed that Calculus II and Circuits I have a similar correlation coefficient between the college factor analysis and high school factor analysis. HS_RANK (High School Rank) had a weak negative correlation with Electric Circuits I grades whereas HS_CLASS_SIZE seems relatively independent. These correlation analyses support using a select group of factors with the logistic regression model. These factors would include HS_RANK, HS_GPA, HS_PERCENTILE, Calculus I \& II grades, ACT composite scores, and Adjusted Cumulative GPA.

Cumulative Effects of Significant Factors

Those factors showing significance in the logistic regression were Calculus I grades, Calculus II grades, Adjusted Cumulative GPA, and HS GPA. The cumulative effects plots of these predictors are shown in Figure 2. 

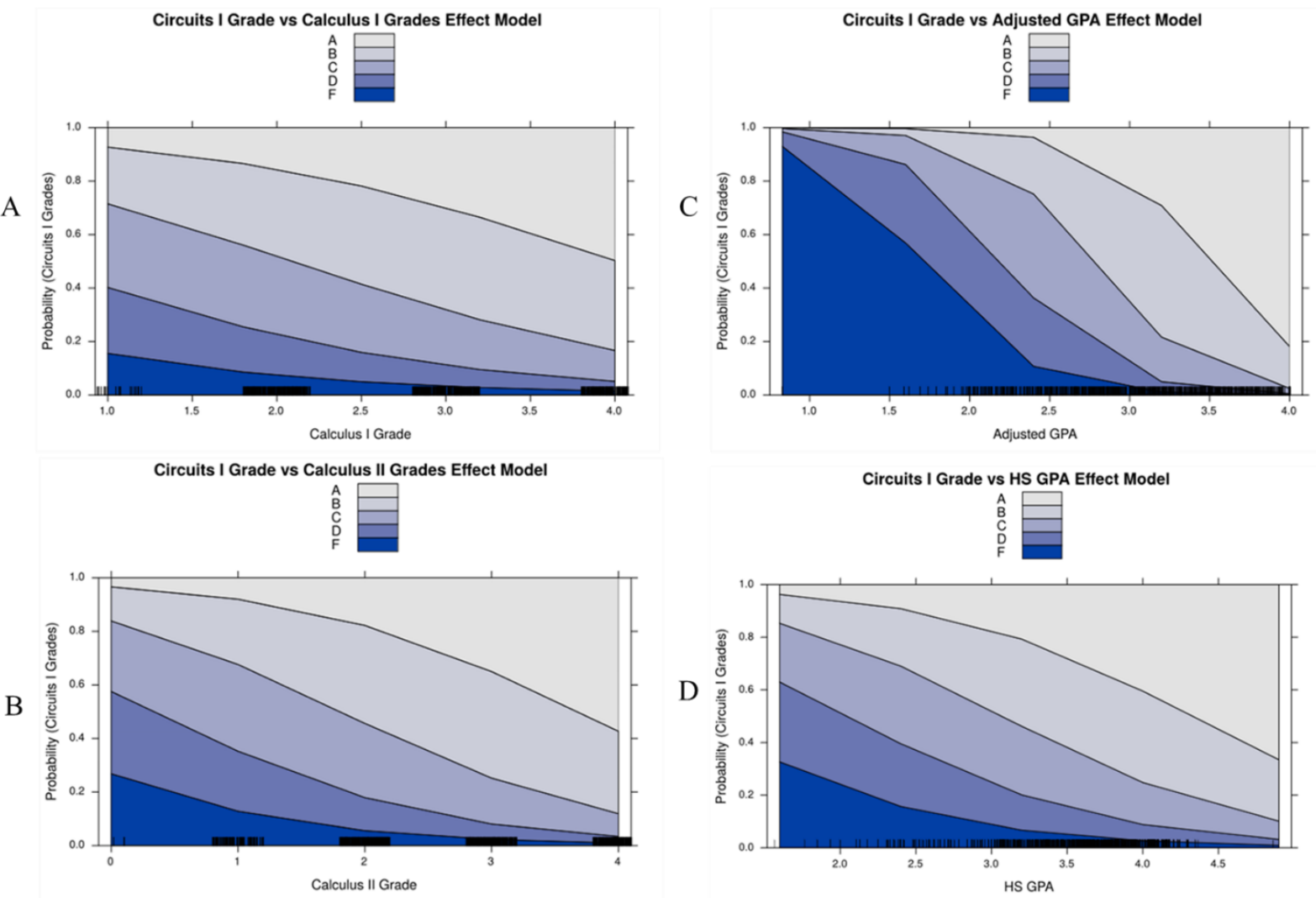

Circuits I Grade vs HS GPA Effect Model

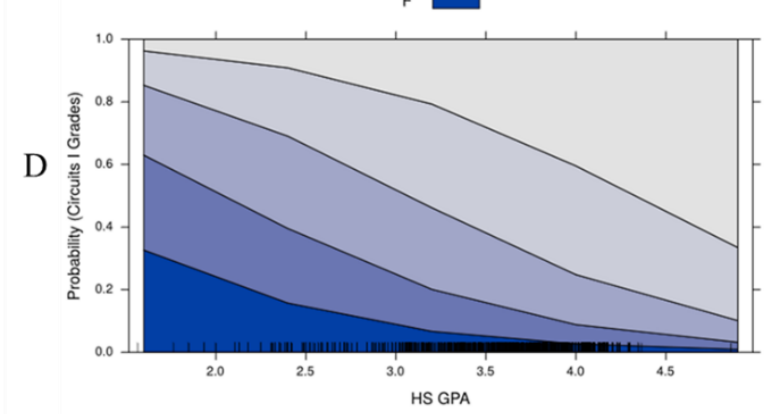

Figure 2. Plots A, B, C, and D are cumulative effects plots that provide the probability of earning a specific grade in Electric Circuits I given a certain performance in the predictor labeled on the horizontal axis.

The four plots in Figure 2, A, B, C, and D all show the probability of a student attaining success in Electric Circuits I on the vertical axis. In this study, success was defined as earning a $\mathrm{C}$ or better in the Electric Circuits I course. The horizontal scale represents the letter grade obtained in either Calculus I (A), Calculus II (B), students Adjusted Cumulative GPA (C), and the students' HS GPA (D) where a 2.0 represents a 'C'. The shaded portions of the plot represent the earned grade in Electric Circuits I. A vertical line can be drawn on the plot and the relative probabilities of a student earning a grade in Circuits I can be determined for a given value of either a Calculus I grade, Calculus II grade, Adjusted GPA, or HS GPA. For instance, for a student who has a 2.0 adjusted cumulative GPA the probabilities a student earns an F, D, C, B or $\mathrm{A}$ is given as $0.36,0.24,0.25,0.12$, and about 0.03 respectively. For a ' $\mathrm{C}$ ' student, their chance of success in Electric Circuits I is approximately $40 \%$ (earning either an A, B, or C) whereas the model predicts approximately a $60 \%$ chance of non-success. For Calculus II as a predictor (plot $\mathrm{B}$ in Figure 2), the chance of Electric Circuits I success is about $65 \%$ and for Calculus I as a predictor (plot A in Figure 2), the chance of success is about $80 \%$. It can be observed that the slope of the delineations between grades in the plots in Figure 2 are higher for the adjusted cumulative GPA whereas the slopes indicate that Calculus I and II grades are less sensitive predictors. This is likely because a typical electrical engineering student taking Electric Circuits 
I would usually do so in the first semester of their sophomore year, after approximately 30 credit hours. Calculus I and II are typically taken in the first year and would represent 8 hours of the average students' course load. Eight credit hours is $26 \%$ of the first-year adjusted cumulative GPA, which would make Calculus I and II grades a significant contributor to adjusted cumulative GPA.

\section{Impact of High School Factors}

High School GPA has been shown to be a better predictor of college performance overall as compared to standardized tests scores [13]. That study examined the four-year graduation rate and cumulative GPA has indicators of success in college. The authors point out that High School GPA is less influenced by a students' economic background as compared to standardized testing scores. Because of available records, our study was not able to incorporate financial or socioeconomic information, so we are unable to clarify the influence of such factors on our main predictors currently. However, the correlation analysis in Figure 1 shows a moderate positive correlation between ACT composite and HS GPA. Our regression analysis indicates that HS GPA is significant with its effects plots showing the probabilities of prediction for Electric Circuits I success in Figure 2 (D). Students had a 50\% chance of success in Electric Circuits I with a 2.0 HS GPA; however, HS GPA was less sensitive a factor than adjusted cumulative college GPA.

\section{Progress Toward Graduation}

Figure 3 shows a students' graduation probability on the vertical axis with the earned Circuits I grade on the horizontal axis. The solid line represents the engineering degree chance of graduation which for the plot of the left is electrical engineering (ELEG) and the plot on the right is for mechanical engineering (MCEG) whereas the dashed line shows the probability the student graduated in an area other than engineering degree program in which they were enrolled at the time of their Electric Circuits I course. The graduation probability for electrical engineering majors as a function of their Circuits I grade shows less probability that other degree types including mechanical engineering. This trend was seen to be less significant for those students earning an $\mathrm{A}$ or a $\mathrm{B}$, and more dramatic for those earning a $\mathrm{C}$ or less. This trend provided the 
justification for establishing a $\mathrm{C}$ or better as success in Circuits $\mathrm{I}$.
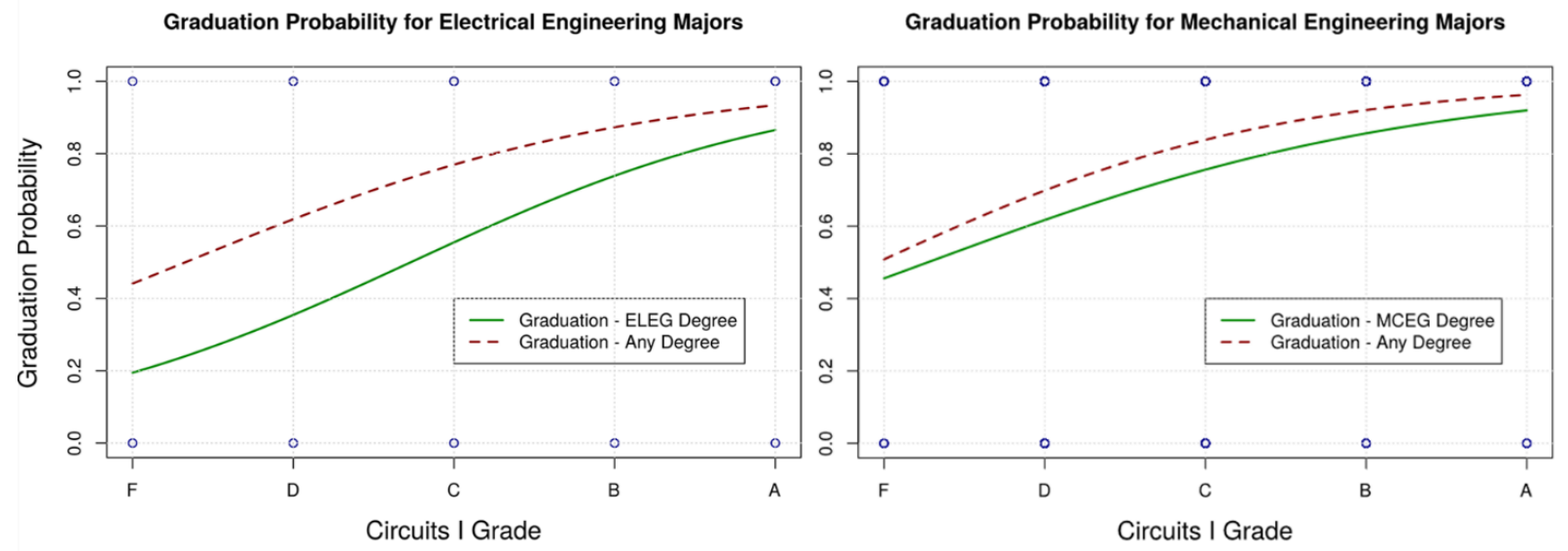

Figure 3. Binary regression analysis output showing the probability of graduation for various college majors in engineering and outside given earned grades in Circuits I.

The earned Circuits I grade appeared to be a more indicative factor of graduation for majors other than electrical engineering. Since Electric Circuits I is a foundational course for electrical engineering majors more so than other disciplines, it was not surprising that it was less sensitive factor for other majors. Figure 4 shows a set of frequency plots that provides information on the total number of years to graduation for both MCEG and ELEG majors following completion of Circuits I.
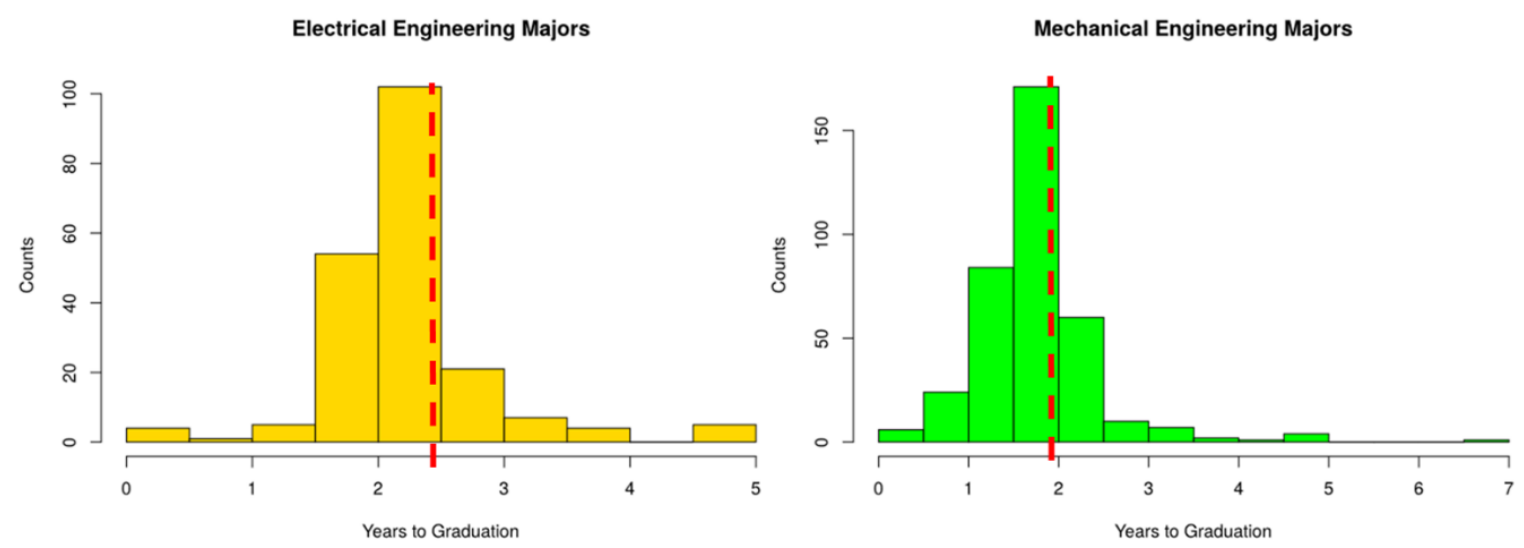

Figure 4. Frequency plot indicating the number of students taking a certain number of years to graduation after taking Electric Circuits I. The vertical red dashed line indicates the average number of years to graduation for each plot.

The electrical engineering (ELEG) majors, on average, take about 2.45 years to reach graduation, whereas the mechanical engineering (MCEG) majors require, on average, 1.97 years to reach graduation which is a result of scheduling it earlier in the electrical engineering curriculum. Electric Circuits I is a prerequisite for Electric Circuits II which, in turn, is a prerequisite for additional courses that ELEG majors are required to take. However, for MCEG majors, no other courses have an Electric Circuits I prerequisite other than Electric Circuits II. Circuits I is 
scheduled in the mechanical engineering curriculum in the spring of their sophomore year which is a semester later than in the electrical engineering curriculum. Because of this, the difference in years to graduation between MCEG and ELEG students might be because the MCEG students are slightly further along in their studies. Taken together in the context of Figure 3 however, this strengthens the idea that tracking a strong predictor of Electric Circuits I success is important to the retention of engineering majors, and to some extent, other majors. Students pursuing an electrical engineering major must make at least a $\mathrm{C}$ to have at least a $50 \%$ chance of attaining their degree in electrical engineering.

\section{Model Verification}

The data was randomly partitioned into a training and testing set and three models were used to predict success in Circuits I ( $\mathrm{C}$ or better) in the testing set based on the coefficients obtained in the training data set. The first model utilized the Adjusted Cumulative GPA as the predictor of success in Circuits I. The second and third models included additional predictor components: Composite ACT in one and Calculus I \& II grades in the other. A receiver operating characteristic (ROC) plot was utilized to evaluate the efficacy of each model. The output of this analysis is shown in Figure 5. The vertical axis provides the sensitivity of the model or how often the regression analysis correctly predicted the correct outcome. The horizontal axis represents 1-Specificity or how often the model incorrectly identified a prediction. Taking the area under the curve (AUC) can provide an estimation of how the model obtained from the regression analysis correctly predicts outcomes. The model performance seems to be optimal or best when Adjusted Cumulative GPA is paired with Composite ACT scores, although the improvement of prediction when considering Composite ACT was not significantly better than just the Adjusted Cumulative GPA alone. Also shown is the ROC characteristic with Adjusted Cumulative GPA and Calculus I and II grades. This plot suggests that Adjusted Cumulative GPA was the best predictor of Electric Circuits I success.

Although the analysis presented here was absent of several non-cognitive factors such as student study habits, student culture, motivation, and self-efficacy that might influence student success in Electric Circuits I and their attainment of a degree, none of the non-cognitive factors that were available (age, gender and ethnicity) were significant predictors of performance in electric circuits I utilizing individual ordered logistic regression analyses. However, the cumulative GPA $(\mathrm{p}<0.001)$ along with Composite ACT $(\mathrm{p}<.01)$ scores represented strong predictive factors in the ordered logistic regression identifying whether students were successful in their Electric Circuits I course. A previous study found that HS GPA was highly predictive of success in their degree in their post-secondary pursuits [9]. The present study suggests that the HS GPA is not as predictive a factor for success in Electric Circuits I as was the cumulative GPA obtained within the first couple of semesters in college. Since Electric Circuits I success was found to be a highly predictive factor for students to complete their degrees in their chosen fields, the ability to identify students who may benefit from additional tutoring or other interventions could be a key element for improving student retention within the program. 
ROC: Success in Circuits I

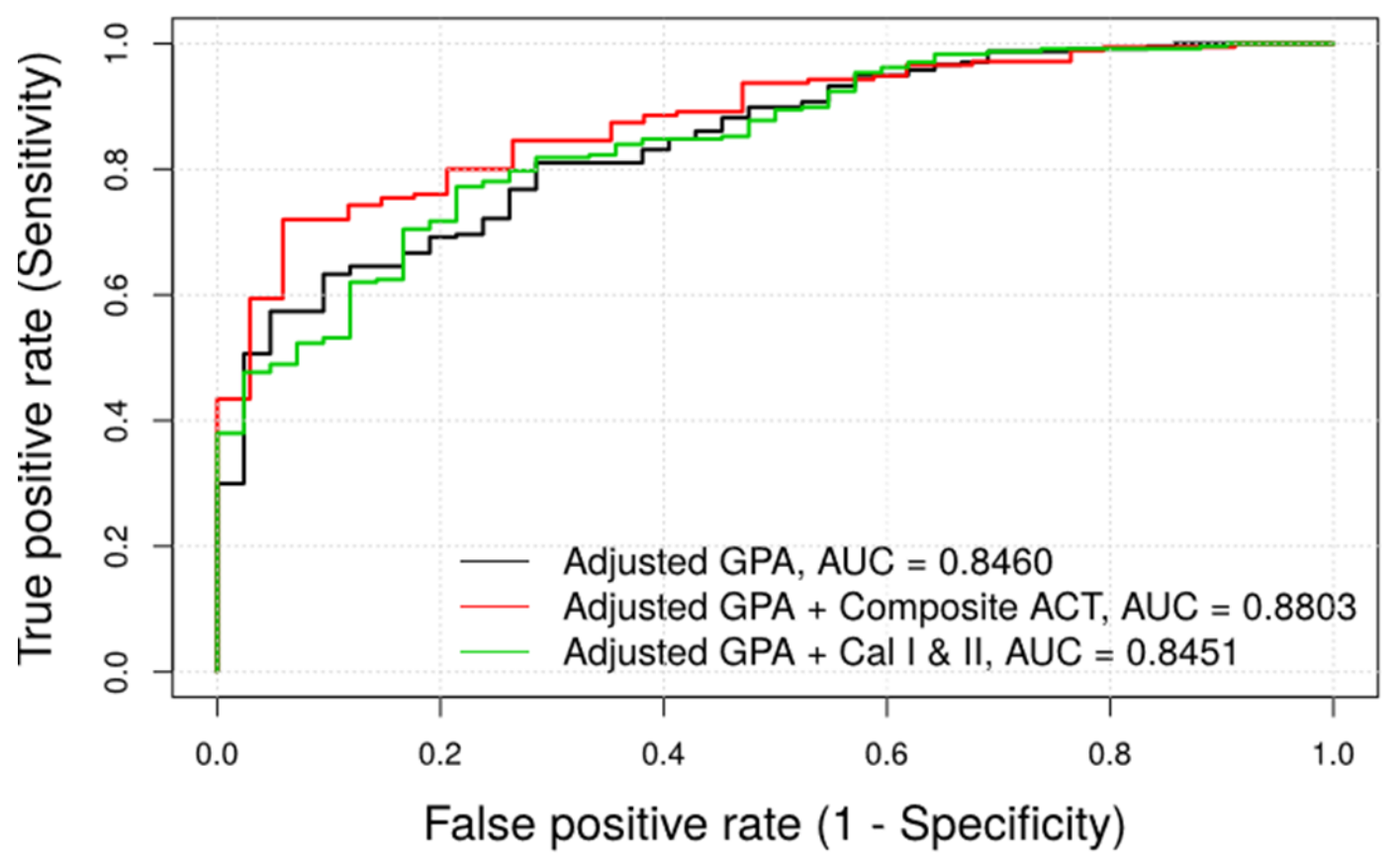

Figure 5. ROC curve providing AUC for quantifying the classifying capability of the regression analysis when using the listed predictors. Perfect classification is $\mathrm{AUC}=1$ whereas a 50\% chance of correct classification is an $\mathrm{AUC}=0.5$.

\section{Conclusion}

The present study utilized correlation analysis, ordinal logistic regression, and generalized linear modeling to examine both cognitive and non-cognitive factors influencing student success in an Electric Circuits I course where success was defined as a ' $C$ ' or better. It was found that Cumulative GPA was the optimal parameter to predict a student's success in Electric Circuits I. It was also observed that retention and completion of the degree in electrical engineering was linked to success in Electric Circuits I. It was not surprising that success in the circuits course was indicative of successful completion of the electrical engineering degree since the majority of subsequent course depend on the knowledge obtained in the Electric Circuits I course.

The non-cognitive factors including age, gender and ethnicity did not play a significant role in the students' success in their Electric Circuits I course. Gender was the closest with a 2.99 (on a 4-point scale) for the female students compared to 2.77 for males. However, it did not reach a $p$ $<0.05$ in a t-test analysis.

This study provided the validation for tracking Electric Circuits I performance and the necessity of early interventions in a student's academic career to increase their probability of degree attainment. The interventions could be extra tutoring, additional faculty support in the form of regular meeting times, or perhaps participation in a peer-based student studying group suggested by the department. 
References

[1] B. F. French, J. C. Immekus, and W. C. Oakes, "An Examination of Indicators of Engineering Students' Success and Persistence," Journal of Engineering Education, vol. 94, no. 4, pp. 419-425, 2005.

[2] A. Karimi and R. Manteufel, "Correlation of Prerequisite Course Grades with Student Performance," in ASEE Annual Conference, Atlanta, GA, 2013.

[3] G. Zhang, T. J. Anderson, M. W. Ohland, and B. R. Thorndyke, "Identifying Factors Influencing Engineering Student Graduation: A Longitudinal and Cross-Institutional Study," Journal of Engineering Education, vol. 93, no. 4, pp. 313-320, 2004.

[4] K. F. Li, D. Rusk, and F. Song, "Predicting Student Academic Performance," in Proceedings of the Seventh International Conference on Complex, Intelligent, and Software Intensive Systems, 2013.

[5] Budny, Dan, Goranka Bjedov, and William LeBold. "Assessment of the impact of the freshman engineering courses." In Proceedings Frontiers in Education 1997 27th Annual Conference. Teaching and Learning in an Era of Change, vol. 2, pp. 1100-1106. IEEE, 1997.

[6] Dekker, Gerben W., Mykola Pechenizkiy, and Jan M. Vleeshouwers. "Predicting Students Drop Out: A Case Study." International Working Group on Educational Data Mining(2009).

[7] Marra, Rose M., Kelly A. Rodgers, Demei Shen, and Barbara Bogue. "Leaving engineering: A multi-year single institution study." Journal of Engineering Education 101, no. 1 (2012): 6-27.

[8] Hackett, Gail, Nancy E. Betz, J. Manuel Casas, and Indra A. Rocha-Singh. "Gender, ethnicity, and social cognitive factors predicting the academic achievement of students in engineering." Journal of Counseling Psychology 39, no. 4 (1992): 527.

[9] Hensel, Robin, JRyan Sigler, and A. Lowery. "AC 2008-2079: BREAKING THE CYCLE OF CALCULUS FAILURE: MODELS OF EARLY MATH INTERVENTION TO ENHANCE ENGINEERING RETENTION." In Proceedings of the 2008 American Society for Engineering Education Annual Conference \& Exposition, Pittsburgh, PA, pp. 22-25. 2008.

[10] Taiyun Wei and Viliam Simko (2017). R package "corrplot": Visualization of a Correlation Matrix (Version 0.84). Available from https://github.com/taiyun/corrplot

[11] Venables, W. N. \& Ripley, B. D. (2002) Modern Applied Statistics with S. Fourth Edition. Springer, New York. ISBN 0-387-95457-0

[12] Blagotić, A. and Daróczi, G. (2014). Rapport: a report templating system. R package version 1.0, URL http://cran.r-project.org/package=rapport

[13] Geiser, Saul, and Maria Veronica Santelices. "Validity of high-school grades in predicting student success beyond the freshman year: High-school record vs. standardized tests as indicators of four-year college outcomes." (2007). 